\title{
Continuous-wave lasing in halide perovskites
}

\author{
Xiaoxia Wang, Xiao Wang and Anlian Pan
}

Continuous-wave (c.w.) lasing means that the laser is operated under continuous optical pumping, which is believed to be a key stepping stone on the path to an electrically pumped laser diode and to applications in high density integrated optoelectronic systems [1]. Achieving continuous-wave lasing was thought to be challenging due to the intrinsic thermal damage and Auger recombination loss, especially in organic semiconductor materials $[2,3]$. In the past two years, with high optical absorption coefficient, low density of defects and especially the suppressed Auger recombination rate, halide perovskites nanostructures are emerging as one of the most promising gain materials for the development of continuous-wave operated lasing, which paves the way for the ultimate goal of electrically pumped laser devices $[4,5]$.

Recently, published in Nature Photonics, Prof. Chris Giebink and co-workers reported the demonstration of optically pumped continuous-wave lasing in methylammonium lead iodide $\left(\mathrm{MAPbI}_{3}\right)$ distributed feedback cavity at low temperature of $100 \mathrm{~K}$ [6]. In this work, the authors explore the gain properties by depositing $\mathrm{MAPbI}_{3}$ film onto a period grating etched into an alumina layer on a high-thermal-conductivity sapphire substrate to avoid the thermal damage (Fig. 1a). In addition, another tricky designing of the continuous-wave laser is the dynamic phase-change process of the $\mathrm{MAPbI}_{3}$ film. As shown in Fig. $1 \mathrm{~b}$, at a substrate temperature of $106 \mathrm{~K}$, the amplified spontaneous emission (ASE) from the $\mathrm{MAPbI}_{3}$ shifts from $750 \mathrm{~nm}$ (orthorhombic phase emission) to $785 \mathrm{~nm}$ (tetragonal phase emission) within several hundred nanoseconds due to the tetragonal phase inclusions formed inside the orthorhombic lattice matrix, presumably because of local excitation light heating. They demonstrated that the photogenerated tetragonal phase inclusions are supposed to function as charge carrier recombination sinks similar to the classical semiconductor quantum wells [7], which reduce the Auger recombination rate and other unexpected non-radiative recombination channels, thus enhancing population inversion and providing optical gain. Notably, the authors also found that lasing from the tetragonal phase inclusions at $106 \mathrm{~K}$ could be sustained for more than $1 \mathrm{~h}$ (Fig. 1c), while lasing death occurs for pure tetragonal-phase $\mathrm{MAPbI}_{3}$ at $T>160 \mathrm{~K}$ [8].

From the intrinsic fundamental physics point of view, further study is required to reveal the mechanism on why this mixed-phase avoids the lasing death phenomenon that occurs in pure tetragonal phase $\mathrm{MAPbI}_{3}$ at $T>160 \mathrm{~K}$. Anyway, in this study, the concept of phase transition and inclusions proposed by Giebink and co-workers provide a useful strategy in the pursuit of an electrically pumped perovskite laser diode, which further reveals that the effort put in diminishing the defect states and other nonradiative recombination channels is critical for achieving c.w. lasing.

Indeed, lasing in a nanoscale oscillator cavity may not require population inversion. With significant excitonpolariton effect in perovskite materials, it is possible to achieve polariton lasing in the perovskite structures especially in a highly confined nanowire cavity with enhanced oscillator strength via reduced mode volume. Very recently, Prof. Xiaoyang Zhu's group from Columbia University has reported c.w. polariton lasing in $\mathrm{CsPbBr}_{3}$ nanowires with low pump intensity [3]. They claimed that both c.w. and pulsed lasing originated from polariton modes based on the cavity modes analysis. The authors also investigated the temperature dependent lasing modes using the Hamiltonian for exciton-photon coupling, which provided further evidences for the low threshold polariton lasing. Although the conversion of polariton lasing to photon lasing is difficult to detect due to degradation of these $\mathrm{CsPbBr}$ nanowires at high pump intensity, the work demonstrates that lead halide perovskite nanowires show promising applications in low

Key Laboratory for Micro-Nano Physics and Technology of Hunan Province, State Key Laboratory of Chemo/Biosensing and Chemometrics, and College of Materials Science and Engineering, Hunan University, Changsha 410082, China

*Email: (anlian.pan@hnu.edu.cn) 
$\mathbf{a}$
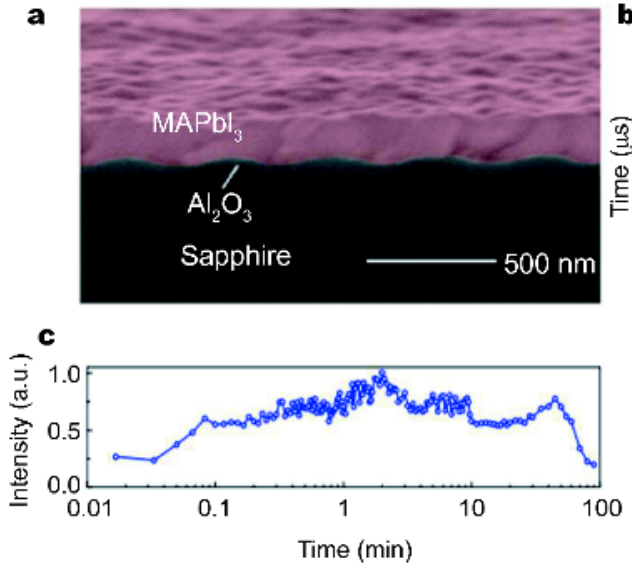

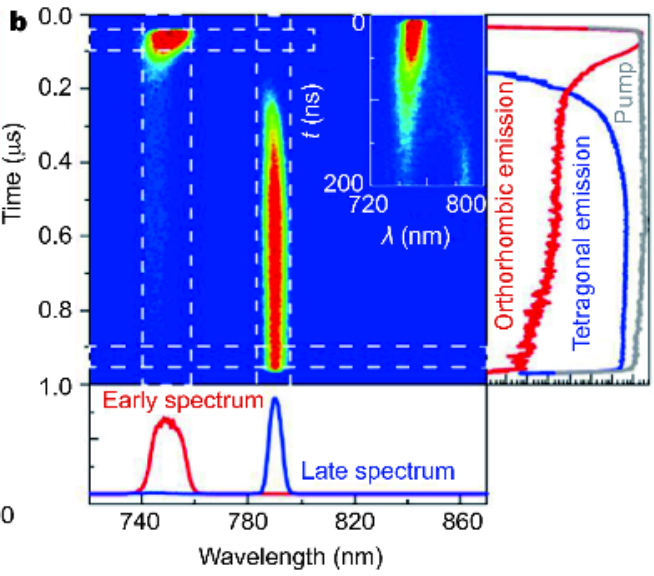

Figure 1 (a) Cross-sectional scanning electron micrograph (SEM) of the second-order DFB laser architecture consisting of a MAPbI ${ }_{3}$ film deposited on an alumina grating on a sapphire substrate. (b) Streak camera image of emission from the $\mathrm{MAPbI}_{3}$ film under 920-ns-long rectangular pump pulse at the substrate temperature of $T=106 \mathrm{~K}$. ASE from the orthorhombic phase of $\mathrm{MAPbI}_{3}$ is observed at early times but evolves within $\sim 200 \mathrm{~ns}$ (see blow-up in the inset) into tetragonal-phase ASE, which is sustained until the end of the pump pulse. (c) Continuous-wave laser emission intensity recorded from a single excitation spot over more than 1 h. Reproduced with permission from Ref. [6], Copyright 2018, Nature Publishing Goup.

power consumption c.w. coherent light sources.

Lastly, these inspiring breakthroughs in c.w. lasing from perovskites undoubtedly represent important steps towards electrically pumped devices. For the prospective of room temperature c.w. lasing, the polariton lasing process in in-organic halide perovskite nanowires is more promising than relying on the phase-change process, e.g. Xiong et al. [9] have reported the experimental realization of room-temperature polariton lasing based on a $\mathrm{CsPCCl}_{3}$ nanoplatelet embedded in a distributed Bragg reflectors (DBR) microcavity. However, according to Zhu's work, room temperature c.w. polariton lasing of the $\mathrm{CsPbBr}_{3}$ nanowires is still facing challenge mainly due to the thermal and light degradation. To this end, the most important task is to improve the nanowire quality since samples with high stabilities would be possible to achieve the conversion of c.w. polariton lasing to photon lasing under high pump density at room temperature. On the other hand, highly confined cavity with enhanced room temperature photon-exciton coupling strength is also worth to develop, which would give rise to high density polaritons for lasing. Basically, the exploitation of the composition dependent exciton-photon coupling strength in $\mathrm{CsPbX}_{3}(\mathrm{X}=\mathrm{Cl}, \mathrm{Br}, \mathrm{I})$ nanowires is also indispensable to give theoretical guidance for room temperature c.w. laser constitution. It shall be expected that these nice studies will inspire efforts towards achieving the ultimate goal of electrically pumped laser devices.

Received 3 April 2018; accepted 4 April 2018;

published online 3 May 2018

1 Sutherland BR, Sargent EH. Perovskite photonic sources. Nat Photonics, 2016, 10: 295-302

2 Fan F, Voznyy O, Sabatini RP, et al. Continuous-wave lasing in colloidal quantum dot solids enabled by facet-selective epitaxy. Nature, 2017, 544: 75-79

3 Evans TJS, Schlaus A, Fu Y, et al. Continuous-wave lasing in cesium lead bromide perovskite nanowires. Adv Opt Mater, 2018, 6: 1700982

4 Zhou H, Yuan S, Wang X, et al. Vapor growth and tunable lasing of band gap engineered cesium lead halide perovskite micro/nanorods with triangular cross section. ACS Nano, 2016, 11: 11891195

5 Zhu H, Fu Y, Meng F, et al. Lead halide perovskite nanowire lasers with low lasing thresholds and high quality factors. Nat Mater, 2015, 14: 636-642

6 Jia Y, Kerner RA, Grede AJ, et al. Continuous-wave lasing in an organic-inorganic lead halide perovskite semiconductor. Nat Photonics, 2017, 11: 784-788

7 Grim JQ, Christodoulou S, Di Stasio F, et al. Continuous-wave biexciton lasing at room temperature using solution-processed quantum wells. Nat Nanotechnol, 2014, 9: 891-895

8 Jia Y, Kerner RA, Grede AJ, et al. Diode-pumped organo-lead halide perovskite lasing in a metal-clad distributed feedback resonator. Nano Lett, 2016, 16: 4624-4629

9 Su R, Diederichs C, Wang J, et al. Room-temperature polariton lasing in all-inorganic perovskite nanoplatelets. Nano Lett, 2017, 17: $3982-3988$ 